\title{
A new algorithm for a CFE-approximated solution of a discrete-time noninteger-order state equation
}

\author{
K. OPRZĘDKIEWICZ ${ }^{1 *}$, R. STANISŁAWSKI ${ }^{2}$, E. GAWIN $^{3}$, and W. MITKOWSKI ${ }^{1}$ \\ ${ }^{1}$ AGH University of Science and Technology, Dept. of Automatics and Biomedical Engineering, 30 Mickiewicza Av., Krakow, Poland \\ ${ }^{2}$ Opole University of Technology, Department of Electrical, Control and Computer Engineering, 76 Prószkowska St., 45-758 Opole, Poland \\ ${ }^{3}$ State Higher Vocational School in Tarnow, Polytechnic Institute, 8 Mickiewicza St., Tarnow, Poland
}

\begin{abstract}
In the paper, a new method for solution of linear discrete-time fractional-order state equation is presented. The proposed method is simpler than other methods using directly discrete-time version of the Grünwald-Letnikov operator. The method is dedicated to use with any approximator to the operator expressed by a discrete transfer function, e.g. CFE-based Al-Alaoui approximation. A simulation example confirms the usefulness of the method.
\end{abstract}

Key words: fractional order calculus, discrete-time noninteger-order state equation, continuous fraction expansion, Al-Alaoui operator.

\section{Introduction}

One of the main areas of application of the fractional order calculus is modeling of processes or plants whose dynamics can not be effectively described by integer-order differential/difference equations. The usefulness of the noninteger-order approach has been presented by many authors, see e.g. [1-6].

Many real applications, to mention model-based control, model-based fault detection, require to implement a noninteger-order model at a digital platform like PLC or FPGA. Known discrete-time state space models of noninteger-order systems are typically based on the Grünvald-Letnikov (GL) definition. An accurate implementation of this model, in particular at the bounded resource platforms, requires a long-length approximation of the GL-based system [7].

The purpose of this paper is to propose a new, discrete-time state space model of a noninteger-order system, constructed with the use of the continuous fraction expansion (CFE) implemented for the Al-Alaoui operator. The use of such an approximant enables to obtain a much more effective model in that the memory length is quite low. This advocates for its use at industrial digital platforms.

The paper is organized as follows. Having recalled the background of the paper in Section 1, Section 2 outlines the fundamentals of fractional-order calculus and introduces a commensurate fractional-order state-space system representation. Section 3 presents various CFE-based approximators to the discretized operator $s^{\alpha}$. Section 4 presents the main results of the paper in terms of solutions to the CFE-aproximated discrete-time noninteger-order state equation. Sections 5 and 6 provide the steady state error and stability analyses for the solution,

*e-mail: kop@agh.edu.pl

Manuscript submitted 2016-12-07, revised 2017-01-29, initially accepted for publication 2017-01-30, published in August 2017. respectively, thus supporting the efficiency of the approach. Example of Section 7 illustrates the usefulness of the methodology and Section 8 summarizes the achievements of the paper.

\section{Preliminaries}

A presentation of elementary ideas begins with a definition of a noninteger-order, integro-differential operator. It is expressed as follows (see for example [8]):

Definition 1. The noninteger-order integro-differential operator is defined as

$$
{ }_{0} D_{t}^{\alpha} f(t)=\left\{\begin{array}{l}
\frac{d^{\alpha} f(t)}{d t^{\alpha}} \quad \alpha>0 \\
1 \quad \alpha=0 \\
\int_{a}^{t} f(\tau)(d \tau)^{-\alpha} \quad \alpha<0
\end{array}\right.
$$

where $a$ and $t$ denote time limits for calculation of the operator, $\alpha \in \mathbb{R}$ denotes the noninteger order of the operation.

Next, an idea of the Gamma (Euler) function (see for example [5]) can be given:

Definition 2. The Gamma function is defined as

$$
\Gamma(x)=\int_{0}^{\infty} t^{x-1} e^{-t} d t .
$$

The fractional-order, integro-differential operator (1) can be described by different definitions, given by Grünvald and Letnikov (GL definition), Riemann and Liouville (RL definition) and Caputo ( $\mathrm{C}$ definition). All these definitions are given 
below. With respect to particular additional assumptions, these definitions can be considered equivalent.

Definition 3. The Grünvald-Letnikov definition of the FO operator $([1,9])$ is as follows:

$$
{ }_{0}^{G L} D_{t}^{\alpha} f(t)=\lim _{h \rightarrow 0} h^{-\alpha} \sum_{j=0}^{\left[\frac{t}{h}\right]}(-1)^{j}\left(\begin{array}{c}
\alpha \\
j
\end{array}\right) f(t-j h) .
$$

In (3), $\left(\begin{array}{l}\alpha \\ j\end{array}\right)$ is a generalization of the Newton symbol into real numbers:

$$
\left(\begin{array}{l}
\alpha \\
j
\end{array}\right)=\left\{\begin{array}{c}
1, \quad j=0 \\
\frac{\alpha(\alpha-1) \ldots(\alpha-j+1)}{j !}, \quad j>0
\end{array}\right.
$$

Definition 4. The Riemann-Liouville definition of the FO operator is as follows:

$$
{ }_{0}^{R L} D_{t}^{\alpha} f(t)=\frac{1}{\Gamma(N-\alpha)} \frac{d^{N}}{d t^{N}} \int_{0}^{\infty}(t-\tau)^{N-\alpha-1} f(\tau) d \tau,
$$

where $N-1<\alpha<N$ denotes the noninteger order of the operation and $\Gamma($.$) is the Gamma function as in (2).$

Definition 5. The Caputo definition of the FO operator is as follows:

$$
{ }_{0}^{C} D_{t}^{\alpha} f(t)=\frac{1}{\Gamma(N-\alpha)} \int_{0}^{\infty} \frac{f^{(N)}(\tau)}{(t-\tau)^{\alpha+1-N}} d \tau .
$$

If the RL or C definitions are considered, the Laplace transform can also be given (see for example [8]) as a generalization of the Laplace transform for the integer-order case:

Definition 6. The Laplace transform for the Riemann-Liouville operator is as follows:

$$
\begin{gathered}
\mathscr{L}\left({ }_{0}^{R L} D_{t}^{\alpha} f(t)\right)=s^{\alpha} F(s), \quad \alpha<0 \\
\left({ }_{0}^{R L} D_{t}^{\alpha} f(t)\right)=s^{\alpha} F(s)-\sum_{k=0}^{n-1} s_{0}^{k} D_{t}^{\alpha-k-1} f(0), \\
\alpha>0, \quad n-1<\alpha<n \in N .
\end{gathered}
$$

Definition 7. The Laplace transform for the Caputo operator is as follows:

$$
\begin{gathered}
\mathscr{L}\left({ }_{0}^{C} D_{t}^{\alpha} f(t)\right)=s^{\alpha} F(s), \quad \alpha<0 \\
\mathscr{L}\left({ }_{0}^{C} D_{t}^{\alpha} f(t)\right)=s^{\alpha} F(s)-\sum_{k=0}^{n-1} s^{\alpha-k-1}{ }_{0} D_{t}^{k} f(0), \\
\alpha>0, \quad n-1<\alpha<n \in N .
\end{gathered}
$$

Consequently, the inverse Laplace transform can be given as (see for example [5] p.15):

$$
\begin{gathered}
{\left[s^{\alpha} F(s)\right]={ }_{0} D_{t}^{\alpha} f(t)+\sum_{k=0}^{n-1} \frac{t^{k-1}}{\Gamma(k-\alpha+1)} f^{(k)}\left(0^{+}\right),} \\
n-1<\alpha<n, \quad n \in \mathbb{Z} .
\end{gathered}
$$

A fractional-order linear state space system is described as:

$$
\begin{aligned}
{ }_{0} D_{t}^{\alpha} x(t) & =A x(t)+B u(t) \\
y(t) & =C x(t)
\end{aligned}
$$

where $\alpha \in(0,1)$ denotes the fractional order of the state equation, $x(t) \in \mathbb{R}^{N}, u(t) \in \mathbb{R}^{L}, y(t) \in \mathbb{R}^{P}$ are the state, control and output vectors, respectively, $A, B, C$ are the state, control and output matrices, respectively.

The discrete time integro-differential operator is based on the (forward) shifted Grünvald-Letnikov definition (3) (see [9]):

Definition 8. The shifted Grünvald-Letnikov definition of the FO operator is as follows

${ }_{0}^{S G L} D_{t}^{\alpha} f(t)=\lim _{h \rightarrow 0} h^{-\alpha} \sum_{j=0}^{\left[\frac{t}{h}+1\right]}(-1)^{j}\left(\begin{array}{c}\alpha \\ j\end{array}\right) f(t-(j-1) h)$.

Next, the (forward) shifted fractional order difference can be defined (see [4]):

Definition 9. The shifted FO difference is as follows:

$$
\Delta^{\alpha} f_{k+1}=\sum_{j=0}^{k+1}(-1)^{j}\left(\begin{array}{c}
\alpha \\
j
\end{array}\right) f_{k-j+1}
$$

and consequently the discrete-time fractional-order linear state space system can be given:

$$
\left\{\begin{array}{l}
\begin{array}{l}
\Delta^{\alpha} x(k+1)=A x(k)+B u(k) \\
x(k+1)=\left(h^{\alpha} A+\alpha I\right) x(k)- \\
\quad-\sum_{j=2}^{k+1}(-1)^{j}\left(\begin{array}{c}
\alpha \\
j
\end{array}\right) x(k-j+1)+h^{\alpha} B u(k) \\
y(k)=C x(k)
\end{array}
\end{array}\right.
$$

\section{Discrete-time approximations of FO operator}

An implementation of operator (1) at each digital platform (PLC, microcontroller) requires to apply an integer-order finite-length discrete-time approximator. The well known approximators are based on PSE (power series expansion) and CFE (continuous fraction expansion). They allow to approximate a noninteger-order element with the use of digital FIR or IIR filters. The PSE approximator is based directly on discrete-time 
version of the GL definition (3) and it has the form of an FIR filter containg only zeros. However its digital, high quality implementation requires to apply a long memory buffer (high order of the filter). The CFE approximator has the form of an IIR filter containing both poles and zeros. It is faster covergent and easier to implement because its order is relatively low, typically not higher that 5 .

The discretization of fractional order element $s^{\alpha}$ can be done with the use of the so called generating function $s \approx \omega\left(z^{-1}\right)$. The new operator raised to the power $\alpha$ has the following form (see for example [10] or [11]:

$$
\begin{aligned}
& \left(\omega\left(z^{-1}\right)\right)^{\alpha}=\left(\frac{1+a}{h}\right)^{\alpha} \operatorname{CFE}\left\{\left(\frac{1-z^{-1}}{1+a z^{-1}}\right)^{\alpha}\right\}_{M, M}= \\
& =\frac{P_{\alpha M}\left(z^{-1}\right)}{Q_{\alpha M}\left(z^{-1}\right)}=\left(\frac{1+a}{h}\right)^{\alpha} \frac{C F E_{N}\left(z^{-1}, \alpha\right)}{C F E_{D}\left(z^{-1}, \alpha\right)}=\frac{\sum_{m=0}^{M} w_{m} z^{-m}}{\sum_{m=0}^{M} v_{m} z^{-m}}
\end{aligned}
$$

where $h$ denotes the sampling time and $M$ is the order of approximation. Numerical values of coefficients $w_{m}$ and $v_{m}$ and various values of the parameter $a$ can be calculated for example with the use of the MATLAB function given in [12].

In Eqn. (14), $a$ is the coefficient depending on an approximation type. For $a=0$ and $a=1$ we obtain the Euler and Tustin approximations, respectively. For $\alpha \in(0,1)$ we arrive at the Al-Alaoui-based approximation, which is a linear combination of the Euler and Tustin approaches. Note that in this case the parameter $a$ in Eqn. (14) is equal to $a=\frac{1-\beta}{1+\beta}$, with $\beta$ being the Al-Alaoui weighting coefficient (see [7, 13]). If the Tustin approximation is considered $(a=1)$ then $C_{D F}\left(z^{-1}, \alpha\right)=C F E_{N}\left(z^{-1},-\alpha\right)$ and the polynomial

Table 1

Coefficients of CFE polynomials $C F E_{N, D}\left(z^{-1}, \alpha\right)$ for Tustin approximation [4]

\begin{tabular}{|l|l|l|}
\hline \multirow{3}{*}{ Order $M=1$} & $w_{m}$ & $v_{m}$ \\
\hline \multirow{5}{*}{$M=3$} & $w_{1}=-\alpha$ & $v_{1}=\alpha$ \\
\cline { 2 - 3 } & $w_{0}=1$ & $v_{0}=1$ \\
\hline \multirow{5}{*}{$M=5$} & $w_{3}=-\frac{\alpha}{3}$ & $v_{3}=\frac{\alpha}{3}$ \\
\cline { 2 - 3 } & $w_{2}=\frac{\alpha^{2}}{3}$ & $v_{2}=\frac{\alpha^{2}}{3}$ \\
\cline { 2 - 3 } & $w_{1}=-\alpha$ & $v_{1}=\alpha$ \\
\cline { 2 - 3 } & $w_{0}=1$ & $v_{0}=1$ \\
\hline & $w_{5}=-\frac{\alpha}{5}$ & $v_{5}=\frac{\alpha}{5}$ \\
\cline { 2 - 3 } & $w_{4}=\frac{\alpha^{2}}{5}$ & $v_{4}=\frac{\alpha^{2}}{5}$ \\
\cline { 2 - 3 } & $w_{3}=-\left(\frac{\alpha}{5}+\frac{2 \alpha^{3}}{35}\right)$ & $v_{3}=-\left(\frac{-\alpha}{5}+\frac{-2 \alpha^{3}}{35}\right)$ \\
\cline { 2 - 3 } & $w_{2}=\frac{2 \alpha^{2}}{5}$ & $v_{2}=\frac{2 \alpha^{2}}{5}$ \\
\cline { 2 - 3 } & $w_{1}=-\alpha$ & $v_{1}=\alpha$ \\
\cline { 2 - 3 } & $w_{0}=1$ & $v_{1}=\alpha$ \\
\hline
\end{tabular}

$C F E_{D}\left(z^{-1}, \alpha\right)$ can be given in the direct form (see [10]). Examples for the polynomial $C_{D} E_{D}\left(z^{-1}, \alpha\right)$ for $M=1,3,5$ are given in Table 1. The approximator using the Muir recursion is presented for example in [14]. The detailed analysis of various forms of the CFE approximators has been given in [7].

\section{Main results}

The approach close to the one proposed here was given in [15], where the use of CFE approximator to solve the discrete time FO state equation was also presented.

Applying the Laplace transform to the continuous-time fractional-order state equation (10) yields:

$$
\begin{aligned}
& s^{\alpha} X(s)-s^{\alpha-1} x_{0}=A X(s)+B U(s) \Longleftrightarrow \\
& \Longleftrightarrow\left(s^{\alpha} I-A\right) X(s)=B U(s)+s^{\alpha-1} x_{0}
\end{aligned}
$$

where $x_{0}$ denotes the initial condition. It is important to notice that it needs to be given for all previous steps (number of steps depends on memory length required by a specific algorithm).

Next we shift to a discrete system by replacing the factor $s^{\alpha}$ by its generating function (backward difference):

$$
\left(I\left(\omega\left(z^{-1}\right)\right)^{\alpha}-A\right) X^{+}\left(z^{-1}\right)=B U^{+}\left(z^{-1}\right)+\sum_{m=-M}^{0} x_{0}(m) .
$$

The generating function is expressed by the CFE approximator (14):

$$
\begin{aligned}
& \left(I \frac{C F E_{N}\left(z^{-1}\right)}{C F E_{D}\left(z^{-1}\right)}-A\right) X^{+}\left(z^{-1}\right)= \\
& =B U^{+}\left(z^{-1}\right)+\sum_{m=-M}^{0} x_{0}(m) .
\end{aligned}
$$

The equation (15) takes the following equivalent form with respect to (14):

$$
\begin{aligned}
& \left(I \sum_{m=0}^{M} w_{m} z^{-m}-\sum_{m=0}^{M} v_{m} z^{-m} A\right) X^{+}\left(z^{-1}\right)= \\
& =\sum_{m=0}^{M} v_{m} z^{-m} B U^{+}\left(z^{-1}\right)+\sum_{m=-M}^{0} x_{0}(m) .
\end{aligned}
$$

Next let us introduce the following matrices $E_{m}$ and $F_{m}$ :

$$
\left\{\begin{array}{l}
E_{m}=w_{m} I_{N \times N}-v_{m} A . \\
F_{m}=v_{m} B . \\
m=0,1, \ldots, M .
\end{array}\right.
$$

After use of (17) the equation (16) can be expressed as:

$$
\sum_{m=0}^{M} E_{m} z^{-m} X\left(z^{-1}\right)=\sum_{m=0}^{M} F_{m} z^{-m} U\left(z^{-1}\right)+\sum_{m=-M}^{0} x_{0}(m)
$$


Equation (18) can be rewritten in the discrete-time domain for the $k$-th time step:

$$
\sum_{m=0}^{M} E_{m} x^{+}(k-m)=\sum_{m=0}^{M} F_{m} u^{+}(k-m)+\sum_{m=-M}^{0} x_{0}(m) .
$$

From (19) the state vector $x^{+}$can be directly calculated as follows:

$$
\begin{aligned}
x^{+}(k) & =-E_{0}^{-1} \sum_{m=1}^{M} E_{m} x^{+}(k-m)+ \\
& +E_{0}^{-1} \sum_{m=0}^{M} F_{m} u^{+}(k-m)+E_{0}^{-1} \sum_{m=-M}^{0} x_{0}(m) .
\end{aligned}
$$

The equation (20) allows to solve the discrete-time FO state equation with the use of the CFE approximant. It has the form of the $M$-th order difference equation. Its solution requires knowing $M$ previous steps of state and control signals.

Further analysis and particular implementations will be easier to perform if (20) will be rewritten as an equivalent 1 st order difference equation of order $M N$. This approach has been applied by many Authors, for example [16-19]. To do it let us introduce extended state and control vectors, denoted by $x_{q}$ and $u_{q}$, respectively:

$$
x_{q}^{+}(k)=\left[\begin{array}{r}
x_{1}^{+}(k) \\
x_{2}^{+}(k) \\
\ldots \\
x_{M}^{+}(k)
\end{array}\right]_{M N \times 1}
$$

where:

$$
\left\{\begin{array}{l}
x_{1}^{+}(k)=x(k) \\
x_{2}^{+}(k)=x(k-1) \\
\cdots \\
x_{M}^{+}(k)=x(k+1-M)
\end{array}\right.
$$

and the control vector is expressed as:

$$
u_{q}^{+}(k)=\left[\begin{array}{r}
u_{1}^{+}(k) \\
u_{2}^{+}(k) \\
\ldots \\
u_{M+1}^{+}(k)
\end{array}\right]_{M+1 \times 1}
$$

where:

$$
\left\{\begin{array}{l}
u_{1}^{+}(k)=u(k) \\
u_{2}^{+}(k)=u(k-1) \\
\cdots \\
u_{M+1}^{+}(k)=u(k-M)
\end{array} .\right.
$$

Equation (25) with respect to $(21-24)$ at the time step $(k+1)$ turns to the following form:

$$
\left\{\begin{array}{l}
x_{q}^{+}(k+1)=A_{q}^{+} x_{q}^{+}(k)+B_{q}^{+} u_{q}^{+}(k) \\
y_{q}^{+}(k)=C_{q}^{+} x_{q}^{+}(k)
\end{array}\right.
$$

where:

$$
\begin{gathered}
A_{q}^{+}=\left[\begin{array}{c}
-E_{0}^{-1} E_{1}, \ldots,-E_{0}^{-1} E_{M} \\
I_{N \times N}, 0,0, \ldots, 0 \\
0, I_{N \times N}, 0, \ldots, 0 \\
\ldots, \ldots, . . ., . . \\
0, \ldots, I_{N \times N}, 0
\end{array}\right]_{M N \times M N} \\
B_{q}^{+}=\left[\begin{array}{c}
E_{0}^{-1} F_{0}, \ldots, E_{0}^{-1} F_{M} \\
0,0,0, \ldots, 0 \\
. ., \ldots, . ., . ., . . \\
0,0,0, \ldots, 0
\end{array}\right]_{M N \times M+1} \\
C_{q}^{+}=\left[\begin{array}{c}
C^{+}, 0, \ldots, 0
\end{array}\right]_{N \times M N} .
\end{gathered}
$$

The initial condition for state equation (25) turns to:

$$
x_{q 0}^{+}=\left[\begin{array}{c}
x^{+}(k-1) \\
x^{+}(k-2) \\
\cdots \\
x^{+}(k-M)
\end{array}\right]_{M N \times 1} .
$$

The input signal vector can be obtained as an output from the state space system:

$$
\begin{aligned}
x_{u_{q}}(k+1) & =A_{i} x_{u_{q}}(k)+B_{i} u(k) \\
u_{q}^{+}(k) & =C_{i} x_{u_{q}}(k)+D_{i} u(k)
\end{aligned}
$$

with the system matrices $A_{i} \in \mathfrak{R}^{M \times M}, B_{i} \in \mathfrak{R}^{M \times 1}, C_{i} \in \mathfrak{R}^{(M+1) \times M}$ and $D_{i} \in \mathfrak{R}^{(M+1) \times 1}$ as follows:

$$
A_{i}=\left[\begin{array}{ccccc}
0 & 0 & 0 & \ldots & 0 \\
1 & 0 & 0 & \ldots & 0 \\
0 & 1 & 0 & \ldots & 0 \\
\vdots & \vdots & \ddots & \vdots & \vdots \\
0 & 0 & 0 & \ldots & 0
\end{array}\right], B_{i}=\left[\begin{array}{c}
1 \\
0 \\
0 \\
\vdots \\
0
\end{array}\right],
$$

$$
C_{i}=\left[\begin{array}{ccccc}
0 & 0 & 0 & \ldots & 0 \\
1 & 0 & 0 & \ldots & 0 \\
0 & 1 & 0 & \ldots & 0 \\
\vdots & \vdots & \ddots & \vdots & \vdots \\
0 & 0 & 0 & \ldots & 0
\end{array}\right], D_{i}=\left[\begin{array}{c}
1 \\
0 \\
0 \\
\vdots \\
0
\end{array}\right] \text {. }
$$


Finally, the whole SISO CFE-based state space approximation of (10) can be obtained as a serial connection of the systems (30) and (25):

$$
\begin{aligned}
\underline{x}_{q}^{+}(k+1) & =\underline{A}_{q}^{+} \underline{x}_{q}^{+}(k)+\underline{B}_{q}^{+} u(k) \\
y_{q}^{+}(k) & =\underline{C}_{q}^{+} \underline{x}_{q}^{+}(k)
\end{aligned}
$$

with the system matrices $\underline{A}_{q}^{+} \in \mathfrak{R}^{M(N+1) \times M(N+1)}, \underline{B}_{q}^{+} \in \mathfrak{R}^{M(N+1) \times 1}$ and $\underline{C}_{q}^{+} \in \mathfrak{R}^{N \times M(N+1)}$ as follows:

$$
\begin{aligned}
& \underline{A}_{q}^{+}=\left[\begin{array}{cc}
A_{q}^{+} & B_{q}^{+} C_{i} \\
0 & A_{i}
\end{array}\right]= \\
& =\left[\begin{array}{cccccc}
-E_{0}^{-1} E_{1} & \ldots & E_{0}^{-1} E_{M} & -E_{0}^{-1} F_{1} & \ldots & -E_{0}^{-1} F_{M} \\
I_{N \times N} & \ldots & 0 & 0 & \ldots & 0 \\
\vdots & \ddots & \vdots & \vdots & \ddots & \vdots \\
0 & \ldots & 0 & 0 & \ldots & 0 \\
0 & \ldots & 0 & 1 & \ldots & 0 \\
\vdots & \ddots & \vdots & \vdots & \ddots & \vdots \\
0 & \ldots & 0 & 0 & \ldots & 0
\end{array}\right] \text {, } \\
& \underline{B}_{q}^{+}=\left[\begin{array}{c}
B_{q}^{+} D_{i} \\
B_{i}
\end{array}\right]=\left[\begin{array}{c}
-E_{0}^{-1} F_{0} \\
0 \\
\vdots \\
0 \\
1 \\
0 \\
\vdots \\
0
\end{array}\right] \text {, } \\
& \underline{C}_{q}^{+}=\left[\begin{array}{ll}
C_{q}^{+} & 0
\end{array}\right] .
\end{aligned}
$$

$$
\begin{aligned}
& \lim _{k \rightarrow \infty} y_{q}^{+}(k)=C\left(\frac{\sum_{m=0}^{M} w_{m}}{\sum_{m=0}^{M} v_{m}}-A\right)^{-1} B u_{s s}= \\
& =C\left[-A+\left(\frac{\sum_{m=0}^{M} w_{m}}{\sum_{m=0}^{M} v_{m}}-A\right)^{-1} \frac{\sum_{m=0}^{M} w_{m}}{\sum_{m=0}^{M} v_{m}} A^{-1}\right] B u_{s s} .
\end{aligned}
$$

Taking into account definition (36) and Eqns. (36) and (40) we arrive at (37).

\section{Stability analysis}

A fundamental tool to analyze the stability for noninteger-order continuous-time systems is the Matignon theorem, given for example in [1]. The stability analysis of discrete time approximations to fractional-order systems is usually based on the analysis of stability/instability areas with respect to eigenvalues of the state matrix of the actual system, or poles of its characteristic equation (see e.g. [20-23]). In this paper we will present a similar solution.

Consider the state-space system of (10), with $\lambda_{i}, i=1, \ldots, M$, being the eigenvalues of the state matrix $A$. The system is approximated by the discrete-time state-space model (32) incorporating the CFE-based approximation as in (14). Assume that 1) the approximation $\omega\left(z^{-1}\right)$ of (14) is stable and 2) the term $\omega\left(e^{-j \varphi}\right), \varphi \in[-\pi, \pi]$, constitutes a simply closed curve in the complex plane. Then the stability/instability areas with respect to $\lambda_{i}, i=1, \ldots, M$, are separated from each other by the contour defined as (compare [22]):

$$
S=\left\{\omega\left(e^{-j \varphi}\right), \varphi \in[-\pi, \pi]\right\}
$$

where

$$
\omega\left(e^{-j \varphi}\right)=\frac{\sum_{m=0}^{M} w_{m}\left(e^{-j \varphi}\right)^{m}}{\sum_{m=0}^{M} v_{m}\left(e^{-j \varphi}\right)^{m}} .
$$

The existence of stability areas for CFE-based approximations to fractional-order systems has been proven in [18]. The stability/instability areas for the discrete-time approximator (14) 

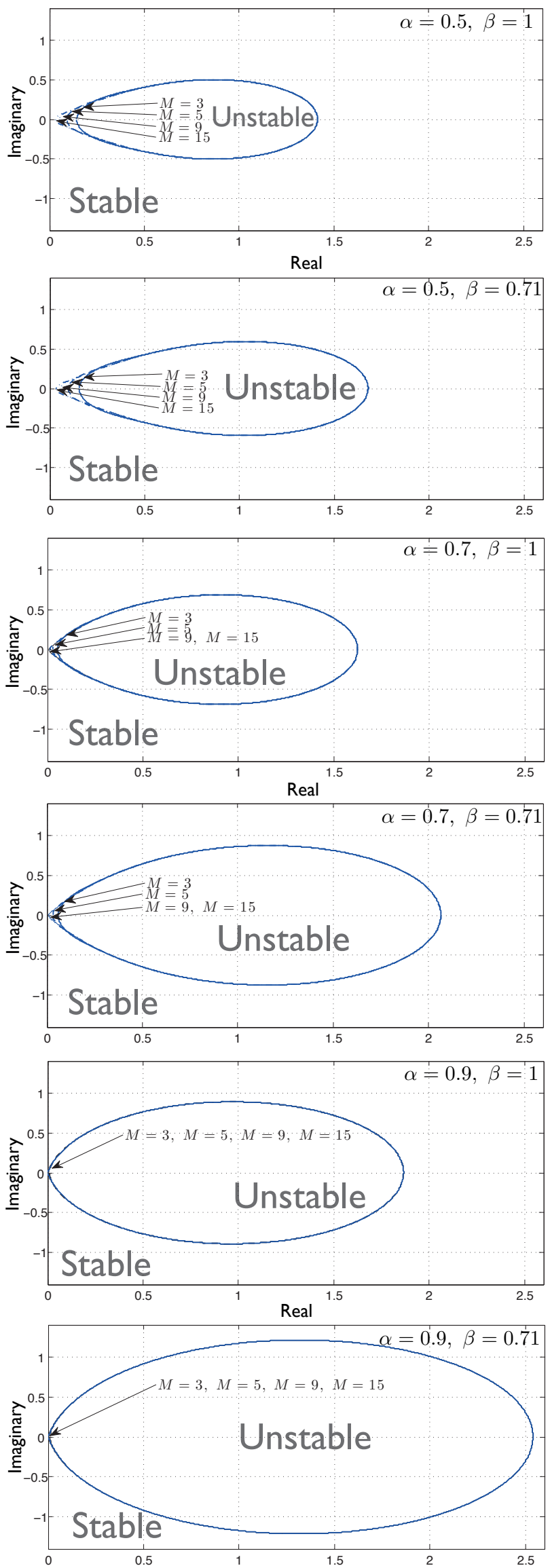

Fig. 1. Stability/instability areas for Al-Alaoui approximator for various fractional orders $\alpha$, weighting coefficients $\beta$ and approximation orders $M$ are presented in Fig. 1.

It can be seen from Fig. 1 that the system is asymptotically stable if and only if the eigenvalues of the matrix $A$ are outside the instability area. The instability areas can be different for various implementation lengths $M$ and different values of $\alpha$ (as can be seen for $\alpha=0.5, \alpha=0.7$ and $\alpha=0.9$ ). For higher values of $\alpha$ the stability areas are quite similar irrespectively of the approximation order $M$. Moreover, Fig. 1 shows that decreasing a value of the weighting coefficient $\beta$ leads to the increase of the instability region. Quite similar results for CFE-based Tustin and PSE-based approximations have been presented in $[21,22]$.

\section{Example}

As an example let us consider the dynamic FO system similar to that analyzed in [20], Ex. 7.3 p. 166, with

$$
\begin{gathered}
A=\left[\begin{array}{ccc}
0 & 1 & 0 \\
0 & 0 & 1 \\
-a_{0} & 0 & 0
\end{array}\right] \quad B=\left[\begin{array}{l}
1 \\
0 \\
0
\end{array}\right] \\
C=\left[\begin{array}{lll}
1 & 0 & 0 \\
0 & 1 & 0 \\
0 & 0 & 1
\end{array}\right] .
\end{gathered}
$$

Let us consider $a_{0}=0.05$ and FO equation with orders of approximation equal to $M=3, M=5$ and non integer orders equal to $\alpha=0.5$ and $\alpha=0.78$.

Let us firstly deal with the homogenous equation and inital conditions in the form:

$$
x_{0}=\left[\begin{array}{ccccc}
1 & 0.5 & 0 & 0 & 0 \\
0 & 0 & 0 & 0 & 0 \\
0 & 0 & 0 & 0 & 0
\end{array}\right] \text {. }
$$

Consider the output equation for $u_{s s}=0$ and $u_{s s}=10$.

Stability/instability areas and system trajectories are presented in Figs. 2 to 9.

For $\alpha=0.5$ the system is stable (Figs. 2 to 5 ), and for $\alpha=0.78$ it is unstable (Figs. 6 to 9 ).

Table 2 shows the results of the calculation of the steady state error (only for $u_{S S}=10$ ).

Table 2

Results for steady state error $\left(u_{s s}=10\right)$

\begin{tabular}{c|c|c|c|c}
\hline & $\begin{array}{c}a=0.5 \\
M=3\end{array}$ & $\begin{array}{c}a=0.5 \\
M=5\end{array}$ & $\begin{array}{c}a=0.78 \\
M=3\end{array}$ & $\begin{array}{c}a=0.78 \\
M=5\end{array}$ \\
\hline \hline$y_{1}$ & 1.1429 & 0.6270 & 0.1290 & 0.0383 \\
\hline$y_{2}$ & -0.0571 & -0.0313 & -0.0064 & -0.0019 \\
\hline$y_{3}$ & -1.4857 & -0.9404 & -0.3250 & -0.1586 \\
\hline
\end{tabular}



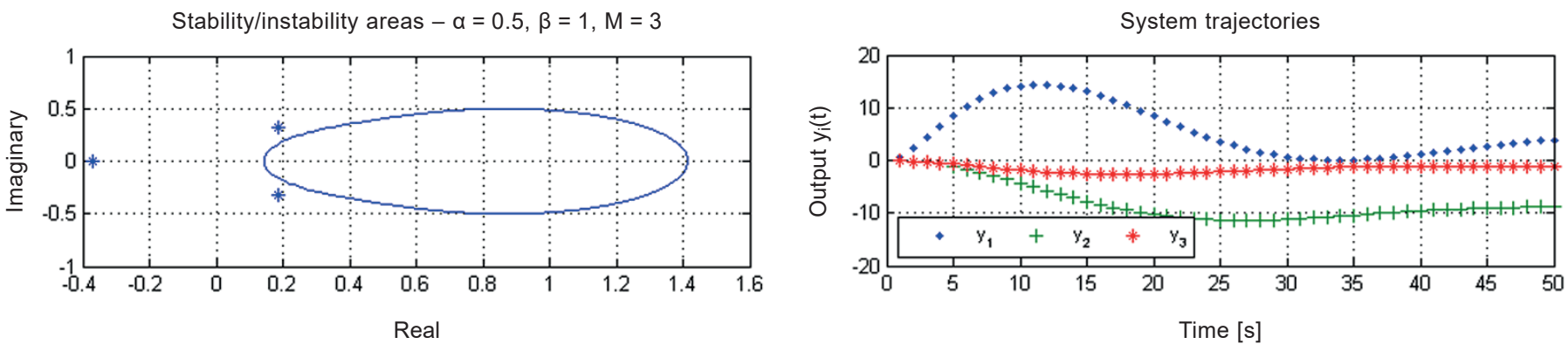

Fig. 2. Stability/instability areas and system trajectories $\alpha=0.5, \beta=1, M=3, u_{s s}=10$
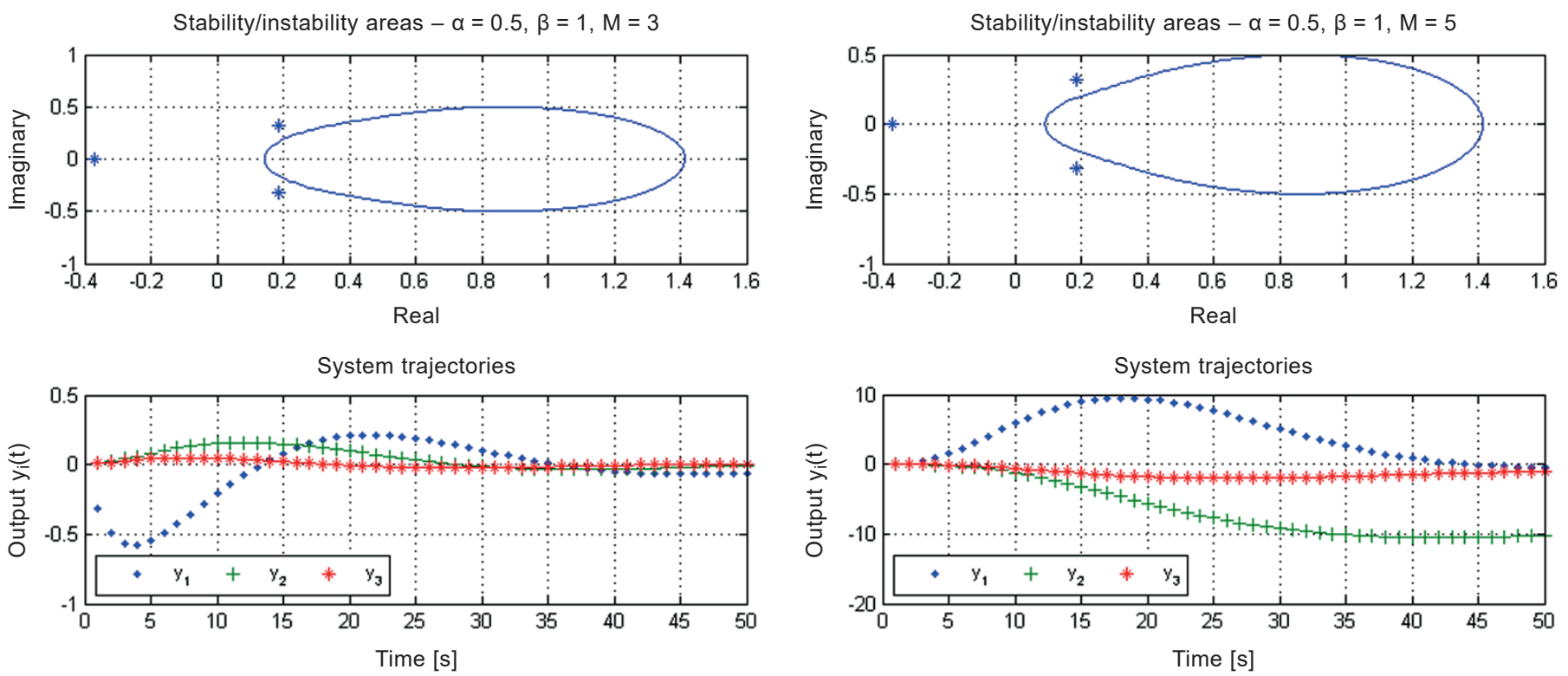

Fig. 3. Stability/instability areas and system trajectories $\alpha=0.5, \beta=1$, $M=3, u_{s s}=0$

Fig. 4. Stability/instability areas and system trajectories $\alpha=0.5, \beta=1$, $M=5, u_{s s}=10$
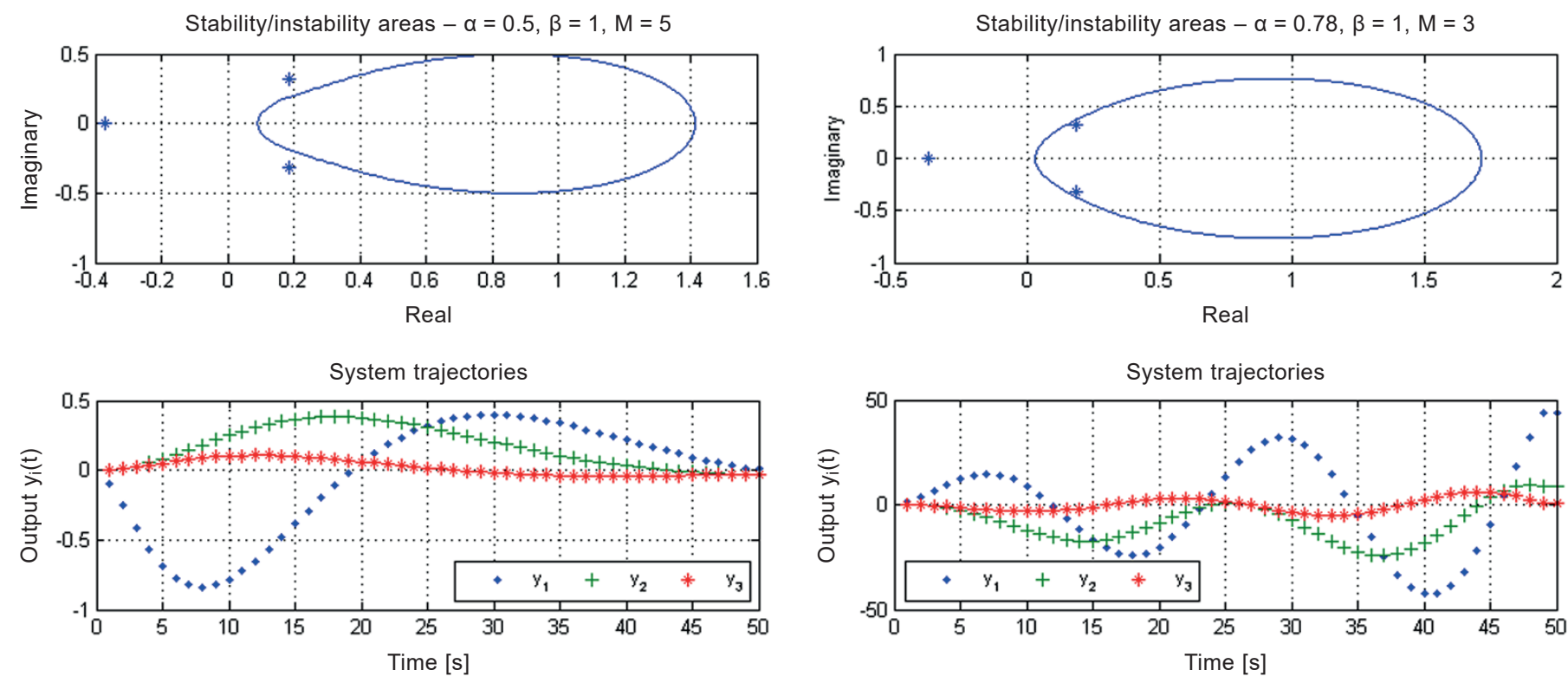

Fig. 5. Stability/instability areas and system trajectories $\alpha=0.5, \beta=1$, $M=5, u_{S S}=0$

Fig. 6. Stability/instability areas and system trajectories $\alpha=0.78$, $\beta=1, M=3, u_{s s}=10$ 

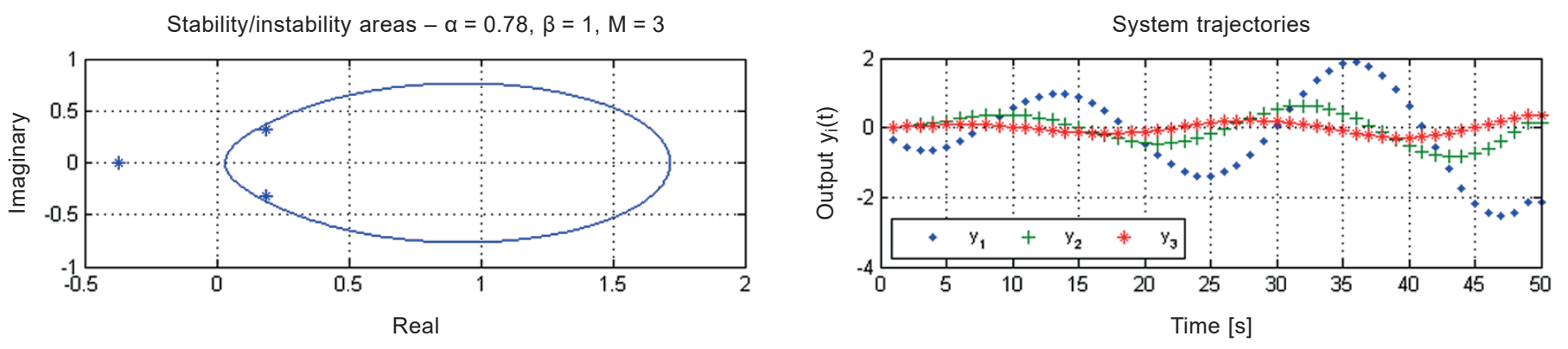

Fig. 7. Stability/instability areas and system trajectories $\alpha=0.78, \beta=1, M=3, u_{s s}=0$
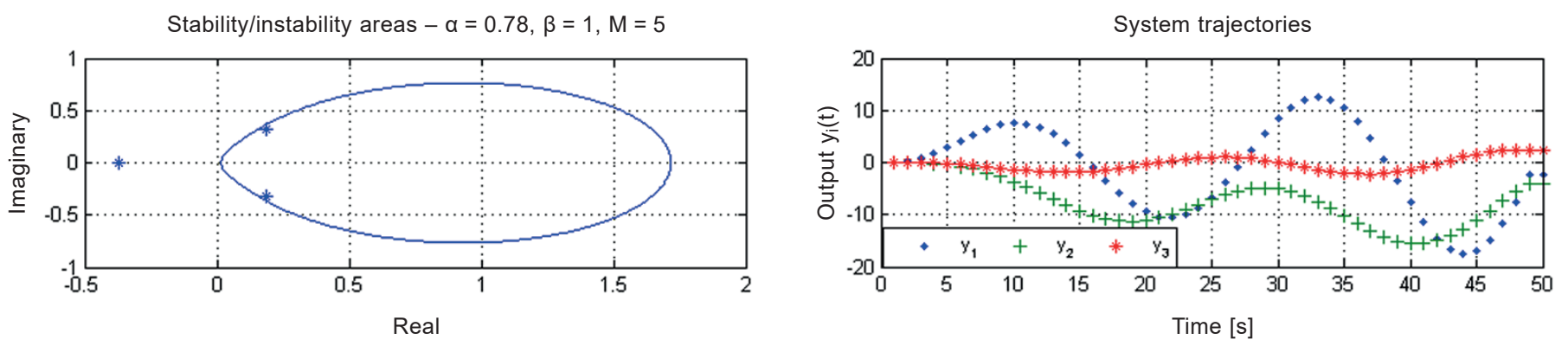

Fig. 8. Stability/instability areas and system trajectories $\alpha=0.78, \beta=1, M=5, u_{S S}=10$
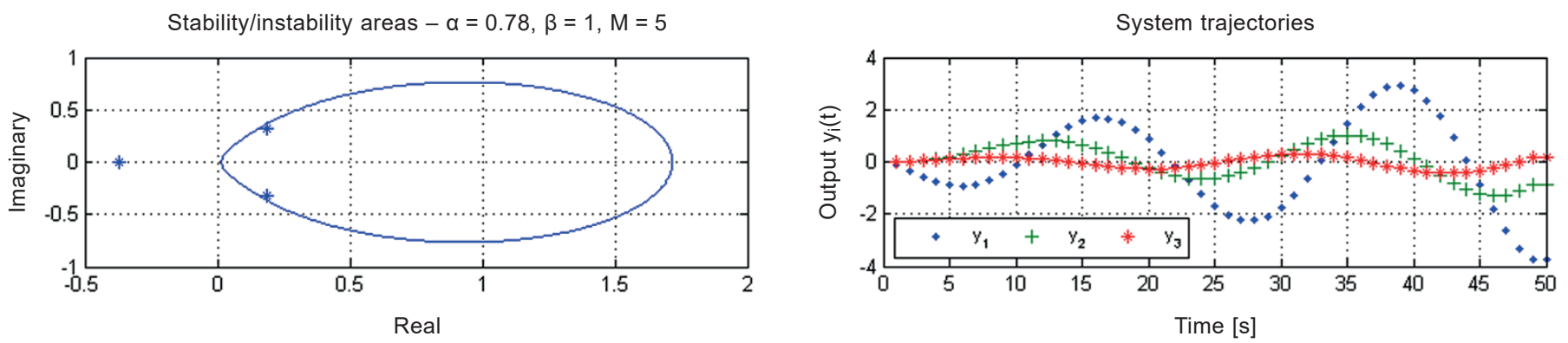

Fig. 9. Stability/instability areas and system trajectories $\alpha=0.78, \beta=1, M=5, u_{S S}=0$

\section{Conclusions}

Final conclusions from the paper can be formulated as follows:

- The proposed new method allows to solve a discrete noninteger-order state equation with the use short-memory discrete-time approximations, for example CFE-based approximation of the Al-Alaoui operator.

- The memory length of the introduced discrete-time model of a noninteger-order state-space system is significantly lower than for other models obtained with the use of the PSE approximation. This is a crucial advantage of the proposed method from the point of view of implementation.

- The stability and steady-state error analyses for the new model can be easily performed.

- Further analysis of the presented model will include its implementation at a digital platform (e.g. PLC) using object-oriented approach recommended by the IEC 61131 standard.

\section{REFERENCES}

[1] R. Caponetto, G. Dongola, L. Fortuna, and I. Petras, (2010), Fractional Order Systems: Modeling and Control Applications, World Scientific Publishing, Singapore, 2010.

[2] S. Das, Functional Fractional Calculus for System Identification and Controls, Springer, Berlin, 2008.

[3] S. Das and I. Pan, Intelligent Fractional Order Systems andControl. An Introduction, Springer, Heidelberg, 2013.

[4] A. Dzieliński, D. Sierociuk, and G. Sarwas, "Some applications of fractional order calculus", Bull. Pol. Ac.: Tech. 58 (4), 583-592 (2010).

[5] T. Kaczorek and K. Rogowski, Fractional Linear Systems and Electrical Circuits, Bialystok University of Technology, Bialystok, 2014.

[6] K. Oprzędkiewicz and E. Gawin, "Non integer order, state space model for one dimensional heat transfer process", Archives of Control Sciences 26 (2), 261-275 (2016). 
[7] R. Stanisławski, K.J. Latawiec, and M. Łukaniszyn, “A comparative analysis of Laguerre-based approximators to the Grünwald-Letnikov fractional-order difference", Mathematical Problems in Engineering 2015, Article ID 512104 (2015).

[8] T. Kaczorek, Selected Problems in Fractional Systems Theory, Springer-Verlag, Berlin, 2011.

[9] P. Ostalczyk, "Equivalent descriptions of a discrete-time fractional-order linear system and its stability domains", International Journal of Applied Mathematics and Computer Science 22 (3), 533-538 (2012).

[10] Y.Q. Chen and K.L. Moore, "Discretization schemes for fractional-order differentiators and integrators", IEEE Transactions on Circuits and Systems I: Fundamental Theory and Applications 49 (3), 363-367 (2002).

[11] I. Petras, "Fractional order feedback control of a DC motor", Journal of Electrical Engineering 60 (3), 117-128 (2009).

[12] I. Petras, http: //people.tuke.sk/igor.podlubny/USU/matlab/petras/dfod2.m.

[13] M.A. Al-Alaoui, "Novel digital integrator and differentiator", Electronics Letters 29 (4), 376-378 (1993).

[14] B.M. Vinagre, Y.Q. Chen, and I. Petras, "Two direct Tustin discretization methods for fractional-order differentiator-integrator", Journal of the Franklin Institute 340, 349-362 (2003).

[15] L. Dorcak, I. Petras, I. Kostial, and J. Terpak, "Fractional-order state space models", Proc. International Carpathian Control Conference, ICCC 2002, 193-198 (2002).
[16] J. Hammer, "Non-linear systems: Stability and rationality", Int J. of Control 39, 11-35 (1984).

[17] Ü. Kotta, A.S.I. Zinober, and P. Liu, "Transfer equivalence and realization of nonlinear higher order input-output difference equations", Automatica 37 (11), 1771-1778 (2001).

[18] Ü. Kotta, E. Pawłuszewicz, and S. Nomm, "Generalization of transfer equivalence for discrete-time non-linear systems: Comparison of two definitions", Int. J. of Control 77 (8), 741-747 (2004).

[19] R.K. Pearson and Ü. Kotta, "Nonlinear discrete-time models: state-space vs. I/O representations", Journal of Process Control 14 (5), 533-538 (2004).

[20] P. Ostalczyk, Discrete Fractional Calculus. Applications in Control and Image Processing, World Scientific Publishing, Singapore, 2016.

[21] M. Siami, M.S. Tavazoei, and M. Haeri, "Stability preservation analysis in direct discretization of fractional order transfer functions”, Signal Processing 9 (2011), 508-512 (2011).

[22] R. Stanisławski and K.J. Latawiec, "Stability analysis for discrete- time fractional-order LTI state-space systems. Part I: New necessary and sufficient conditions for asymptotic stability", Bull. Pol. Ac.: Tech 61 (2), 353-361 (2013).

[23] R. Stanisławski and K.J. Latawiec, "Stability analysis for discrete-time fractional-order LTI state-space systems. Part II: New stability criterion for FD-based systems", Bull. Pol. Ac.: Tech 61 (2), 362-370 (2013). 